\title{
Photon absorption potential coefficient as a tool for materials engineering
}

\author{
Raphael Oluwole Akande $^{1} \cdot$ Emmanuel Oluwole Oyewande $^{1}$
}

Received: 16 September 2015/Accepted: 24 August 2016/Published online: 10 September 2016

(c) The Author(s) 2016. This article is published with open access at Springerlink.com

\begin{abstract}
Different atoms achieve ionizations at different energies. Therefore, atoms are characterized by different responses to photon absorption in this study. That means there exists a coefficient for their potential for photon absorption from a photon source. In this study, we consider the manner in which molecular constituents (atoms) absorb photon from a photon source. We observe that there seems to be a common pattern of variation in the absorption of photon among the electrons in all atoms on the periodic table. We assume that the electrons closest to the nucleus (En) and the electrons closest to the outside of the atom (Eo) do not have as much potential for photon absorption as the electrons at the middle of the atom (Em). The explanation we give to this effect is that the En electrons are embedded within the nuclear influence, and similarly, Eo electrons are embedded within the influence of energies outside the atom that there exists a low potential for photon absorption for them. Unlike En and Eo, Em electrons are conditioned, such that there is a quest for balance between being influenced either by the nuclear force or forces external to the atom. Therefore, there exists a higher potential for photon absorption for Em electrons than for En and Eo electrons. The results of our derivations and analysis always produce a bell-shaped curve, instead of an increasing curve as in the ionization energies, for all elements in the periodic table. We obtained a huge data of PAPC for each of the several materials considered. The
\end{abstract}

Raphael Oluwole Akande

raphaelakande@myashiritycode.com

Emmanuel Oluwole Oyewande oe.oyewande@mail1.ui.edu.ng

1 Theoretical Physics Group, University of Ibadan, Ibadan, Oyo, Nigeria point at which two or more PAPC values cross one another is termed to be a region of conflicting order of ionization, where all the atoms absorb equal portion of the photon source at the same time. At this point, a greater fraction of the photon source is pumped into the material which could lead to an explosive response from the material. In fact, an unimaginable and unreported phenomenon (in physics) could occur, when two or more PAPCs cross, and the material is able to absorb more than that the photon source could provide, at this point. These resulting effects might be of immense materials engineering applications.

Keywords Papc $\cdot$ Ionization $\cdot$ Photon $\cdot$ Molecular interactions $\cdot$ Energy absorption

\section{Introduction}

The photon absorption potential coefficient (PAPC) is the tool we have proposed to better represent the instantaneous status of the atom, while interacting with its environment, as regards its ability to absorb energy. Since the ionization energies are based on the non-interacting atomic energy levels, they are a subset of the actual (effective) energy an atom is interacting with $[1,2]$. For instance, according to the ionization energy principles, two hydrogen atoms are the same even if one is in a supercooled environment and the other in a plasma. Anyway, since the hydrogen energy level system is less complicated, one may agree but this is not always the case with heavier atoms. In the work of Giovanni et al. [3], there is a form of chemical reaction that takes place when some polymers are exposed to $\mathrm{KeV}$ energies. It was also reported that there are interface and interdiffusion effects in systems, such as $\mathrm{Co} / \mathrm{Si}, \mathrm{MgO}$, and $\mathrm{TiO}_{2}$ when exposed to photon beams $[4,5]$. Brumer and 
Shapiro [6] studied and reported the relatively small response of biological molecules to photon absorption. The literatures reviewed confirm the existence of some unique responses of molecules to photon absorption but did not associate their findings to the ionization patterns of the molecules studied. They were only interested in their responses to those photon exposure. On the contrary, our focus is to study (theoretically) the distribution, sharing and response of atoms, within a molecule, to photon exposure. The response of atoms to photon absorption will, therefore, be a function of order of ionizations within the molecule. The molecule is a mixture of competing ionizations, and the order of ionization, within the molecule, is of interest leading the way to understanding the nature of responses different molecules exhibit when exposed to photon.

While we do not condemn the essence of ionization energy [7], we have chosen to derive the PAPC to enable scientists determine/simulate the response of an atom, in an environment made up of different (equally absorbing) atoms, to photon absorption. We have observed that electrons seem to keep absorbing energy at a rate that depends on the total energy from the source. That means (as it is already well known that), two different molecules (in the same environment) will absorb photon at different rates depending on the PAPCs of their constituent atoms. Similarly, a material will absorb photon at different rates when in different (hot and cold) environments. Therefore, a material made of such different molecules, either by accident or design, would have a variety of responses to photon absorption. In fact, we found out that a unique material could be formed if the PAPCs of most or all of its constituent atoms, at an instance of energy, cross one another. The point at which two or more PAPC values cross one another is termed to be a region of conflicting order of ionization, where all the atoms absorb a portion of the photon source at the same time. At this point, a greater fraction of the photon source is pumped into the material leading to an explosive response from the material.

\section{Methods}

\section{The importance of a PAPC}

We have observed that if ionization energy increases down to the inner most electron, then the absorption of photon decreases down to the inner most electrons as well [9]. Therefore, this clearly shows that while ionization energies are the energies binding the electrons to the nucleus, they are not, necessarily, the energies absorbed by the electrons. The little spikes at mid-points of all PAPC is due to some irregularities in the atomic system which is more for the even $z$ atoms than the odd $z$ atoms, as our results show. We also note that while the inner-most electron is purely not interacting with the outside of the atom, the outer-most electron is heavily interacting with the outside of an atom. Hence, both of them will have the lowest PAPCs than other electrons in the atom. That is why we have that bell-shaped curve.

The PAPC is given as:

$$
\begin{aligned}
A_{c} & =\frac{1}{2 z}\left\{\ln \left(\frac{1+z^{-}}{1-z^{-}}\right)+\ln \left(\frac{1+z^{+}}{1-z^{+}}\right)\right\} \\
& =\frac{1}{2 z}\left\{\ln \left(\frac{1+z^{-}+z^{+}+z^{-} \times z^{+}}{1-z^{-}-z^{+}+z^{-} \times z^{+}}\right)\right\} .
\end{aligned}
$$

Equation (1) can also be written as [8]:

$A_{c}=\frac{1}{z}\left(\tanh ^{-1}\left(z^{-}\right)+\tanh ^{-1}\left(z^{+}\right)\right)$,

where $z^{-}$is given as:

$$
z^{-}=\frac{z_{v}^{-}}{\left|z-z_{v}^{-}\right|}
$$

and $z^{+}$is given as:

$$
z^{+}=\frac{z_{v}^{+}}{\left|z-z_{v}^{+}\right|}
$$

where $z_{v}^{+}$and $z_{v}^{-}$are the extra subtracted and added electrons of the neutral electrons of the atom in question (Fig. 1).

Take for instance, the $A_{c}$ of an atom like $\mathrm{Na}$ is will be given as follows:

- $\quad$ For the first ionization, we have: $z_{v}^{+}=\frac{12}{|11-12|}=12$ and $z_{v}^{-}=\frac{10}{|11-10|}=10 . A_{c}=0.0167148$.

- For second ionization, we have: $z_{v}^{+}=\frac{13}{|11-13|}=6.5$ and $z_{v}^{-}=\frac{9}{|11-9|}=4.5 . A_{c}=0.0346427$.

- For the third ionization, we have: $z_{v}^{+}=\frac{14}{|11-14|}=$ 4.66667 and $z_{v}^{-}=\frac{8}{|11-8|}=2.66667 . A_{c}=0.0556262$.

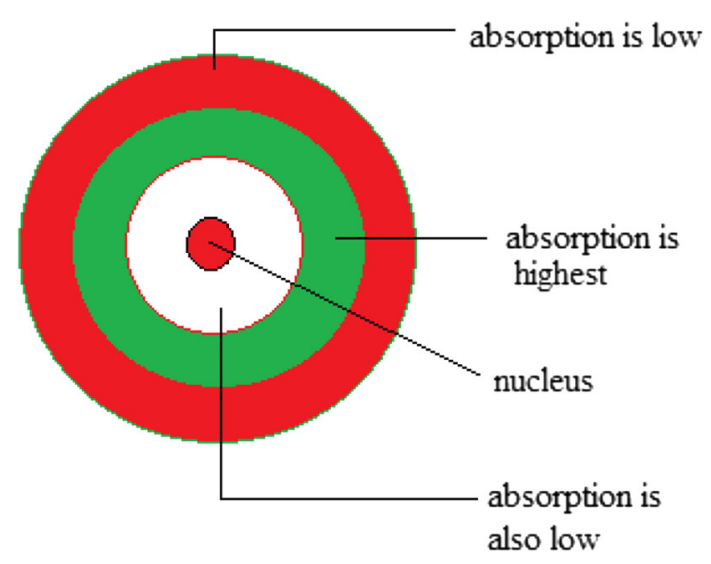

Fig. 1 Illustrating the need for the PAPC and the origin of the PAPC curve 
We could also have the rate of diffusion of PAPC as $A_{r}=A_{c} \frac{c}{R}$, where $c$ is the speed of the transported photon and $R$ is the increasing distance of the transporting energy from the landing site of the foreign particle (Figs. 2, 3).

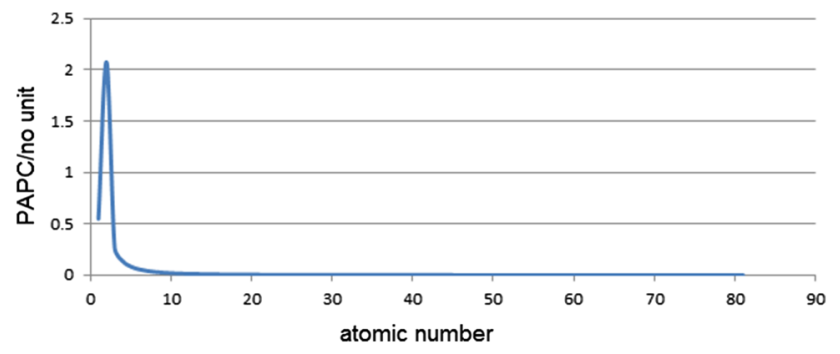

Fig. 2 This is the first PAPCs for each of the first 80 elements

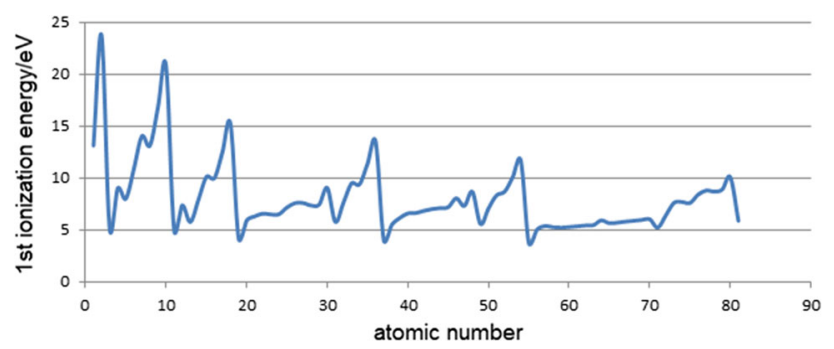

Fig. 3 First ionization energies of the first 80 elements

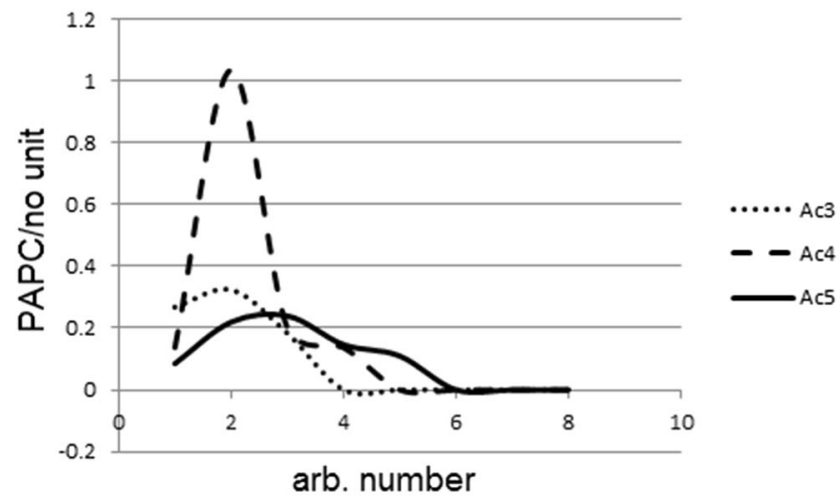

Fig. 4 PAPCs $\left(A_{c}\right)$ for atoms with atomic numbers $z=3-8$
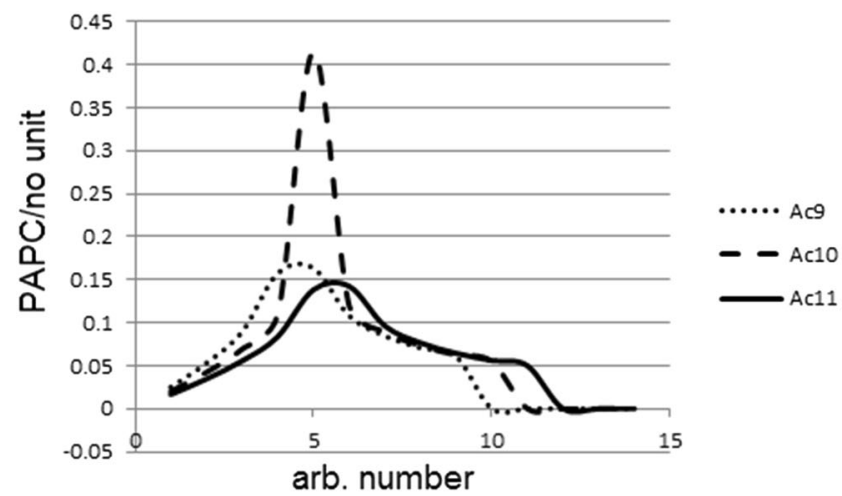

Fig. 5 PAPCs $\left(A_{c}\right)$ for atoms with atomic numbers $z=9-14$

\section{Generating a PAPC genome for materials}

In the following figures, we show our findings per the usefulness of PAPC. From each of Figs. 4, 5, 6, 7, 8, 9, 10, $11,12,13,14,15$, and 16, we can see that in Fig. 4, where $z=4$, beryllium is the element with the most potential for photon absorption, followed by $z=6$, carbon, and then $z=8$, oxygen. This, even-odd $z$, repetition is the same for each group where for each group (represented by each figures), we have: for the even $z$, the $z=n, z=n+2$, and $z=n+4$, (where $n=z+1$ for each figure) are the ones with the most potential for photon absorption, respectively. Similarly, for the odd $z$, the $z=n, z=n+2$, and $z=n+4$, (where $n=z$ for each figure) are the ones with the most potential for photon absorption, respectively. This trend is understandable from the generating function for $A_{c}$ and can be interpreted to mean that all atoms of all elements originate from the same pattern, with respect to PAPC model. The rippled tail at the latter parts of each figure is due to the fact that we have set the remains of the shorter $z$ to 0 . In the following PAPC plots, the $x$-axis is the number of electrons, and it also means the energy of that number of electrons.

The comparison in Table 1, going by the differences in the PAPCs, suggests a form of potential for the conflicting
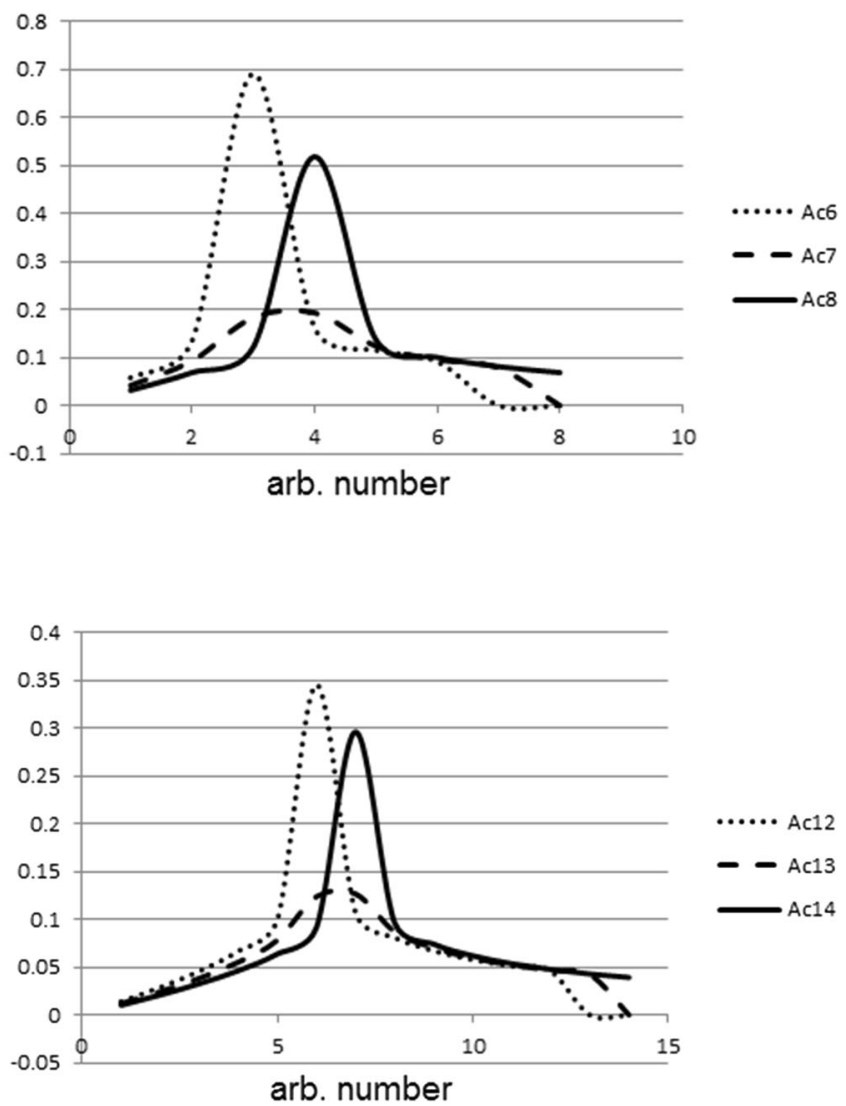


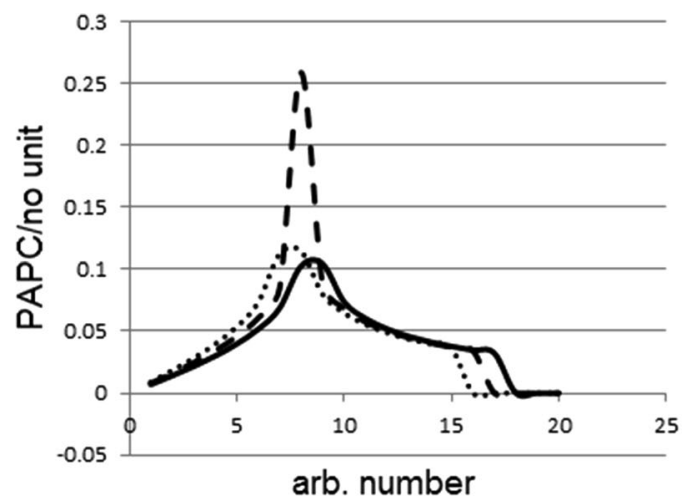

Fig. 6 PAPCs $\left(A_{c}\right)$ for atoms with atomic numbers $z=15-20$

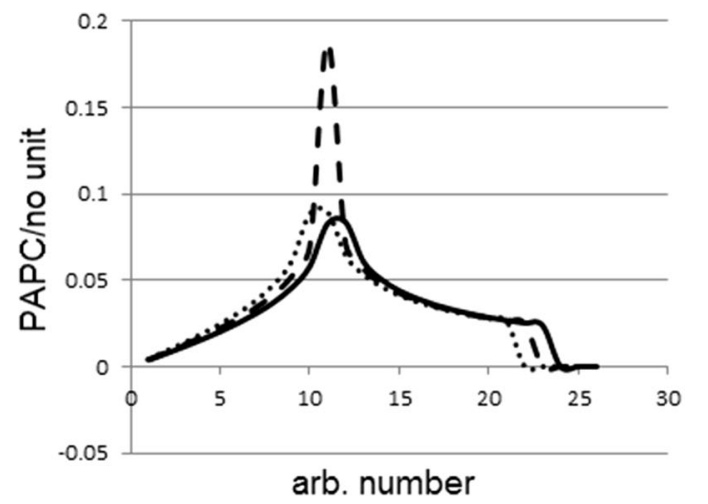

Fig. 7 PAPCs $\left(A_{c}\right)$ for atoms with atomic numbers $z=21-26$
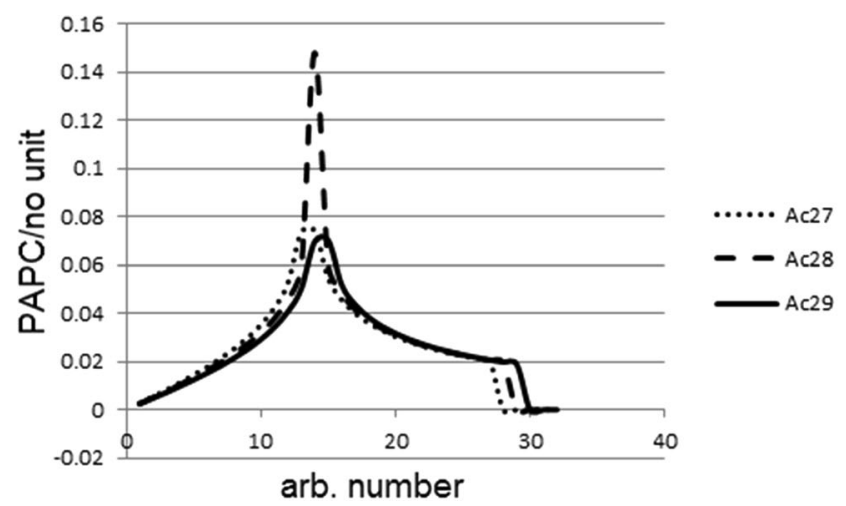

Fig. 8 PAPCs $\left(A_{c}\right)$ for atoms with atomic numbers $z=27-32$

order of ionizations among the atoms grouped together. An unimaginable and unreported phenomenon (in physics) could occur, when two or more PAPCs cross one another, and the material is able to absorb more than the photon source could provide at this point. Therefore, these resulting effects might be of immense materials engineering applications (Figs. 17, 18, 19).
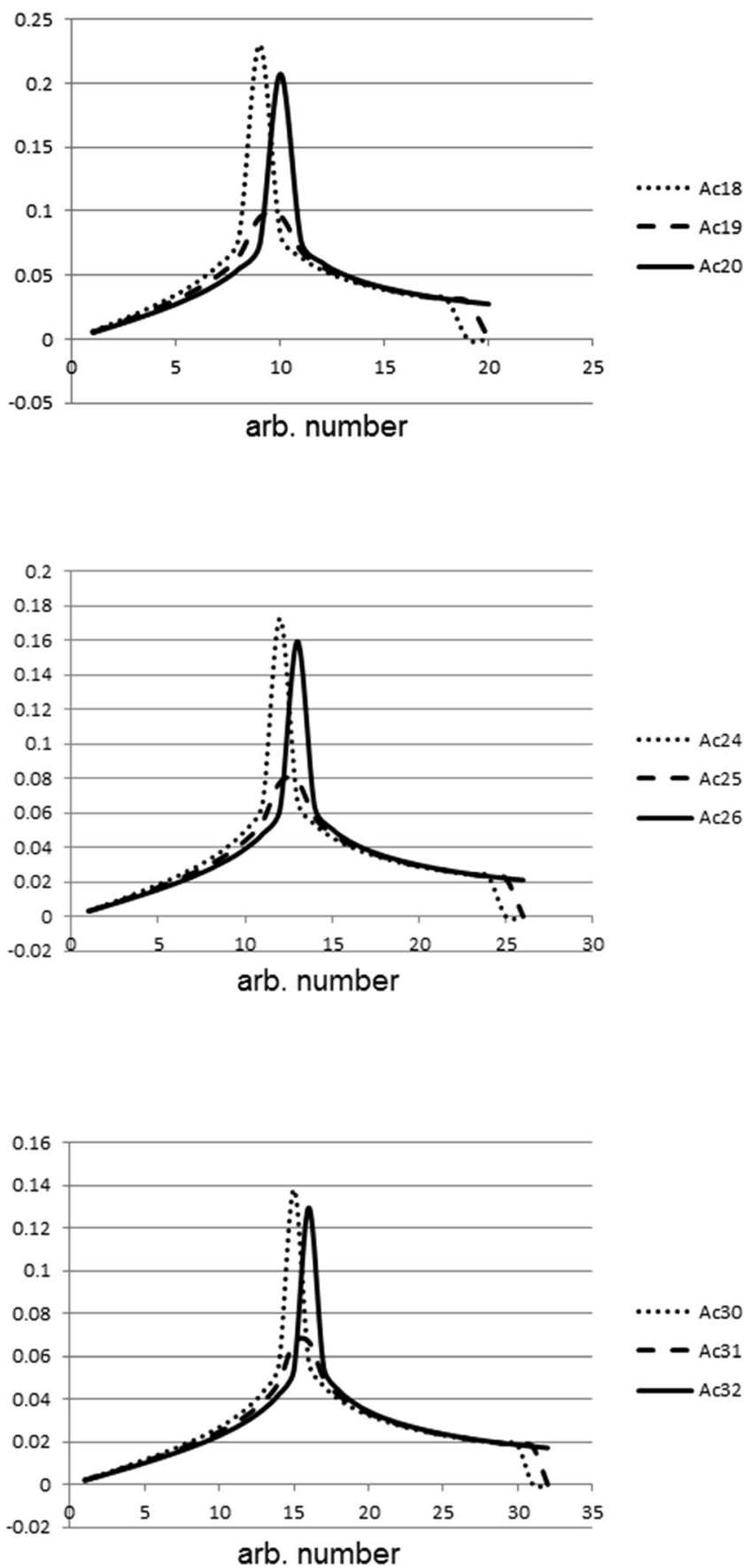

\section{Results and conclusion}

Now, we want to compare the PAPCs of each elements of each group (or figure) as follows in Figs. 4, 5, 6, 7, 8, 9, 10, $11,12,13,14,15$ and 16 and in Table 1 . Thereafter, we intend to compare the PAPC plots of some molecules to explore the pattern they exhibit with respect to their 

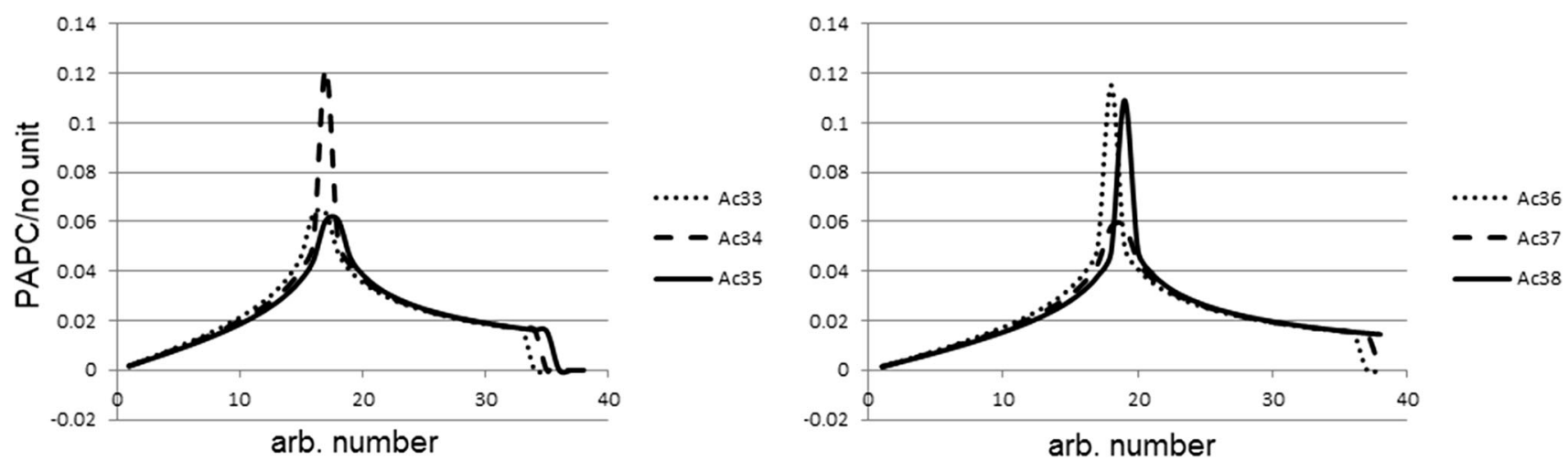

Fig. 9 PAPCs $\left(A_{c}\right)$ for atoms with atomic numbers $z=33-38$
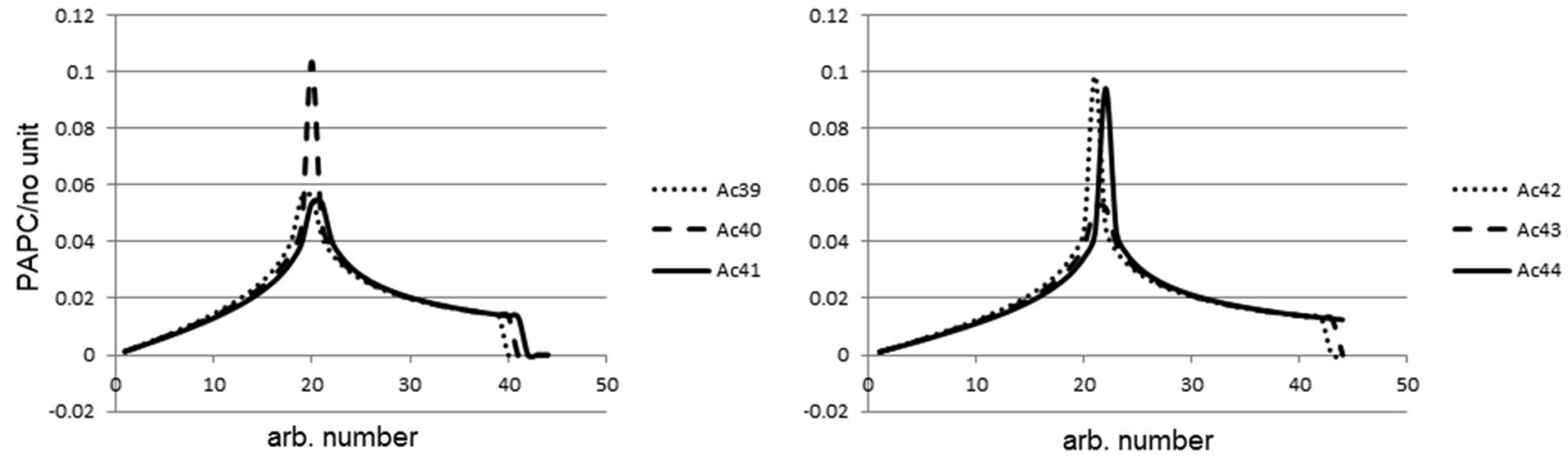

Fig. 10 PAPCs $\left(A_{c}\right)$ for atoms with atomic numbers $z=39-44$
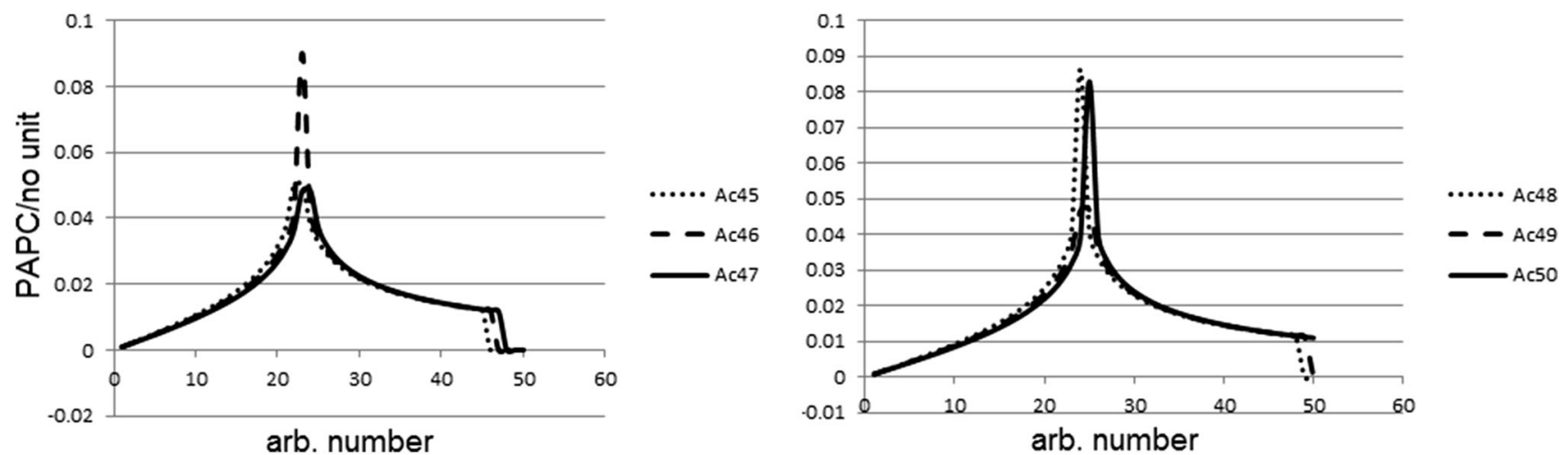

Fig. 11 PAPCs $\left(A_{c}\right)$ for atoms with atomic numbers $z=45-50$

PAPCs. This will lead us to knowing the most possible point of stability, wherein the constituent atoms will be coherent in their photon absorption. Recall that PAPC physically means the fraction of energy obtained from an incoming photon. Therefore, for a group of atoms, i.e., molecules, to be at close PAPC values, it means they will absorb an almost equal amount of the incoming photon. This means that a very reactive material is sensitive to heat

or photon. We shall, therefore, seek a material with a single spot of PAPC for all its component atoms. However, we shall focus on investigating the values at which PAPCs of most materials are at their closest and then provide a meaning for the behavior of such materials. In addition, for cases of more than one spots of PAPCs, we shall report such materials' behavior and try to explain how to artificially make their PAPCs close. Similarly, we shall look 

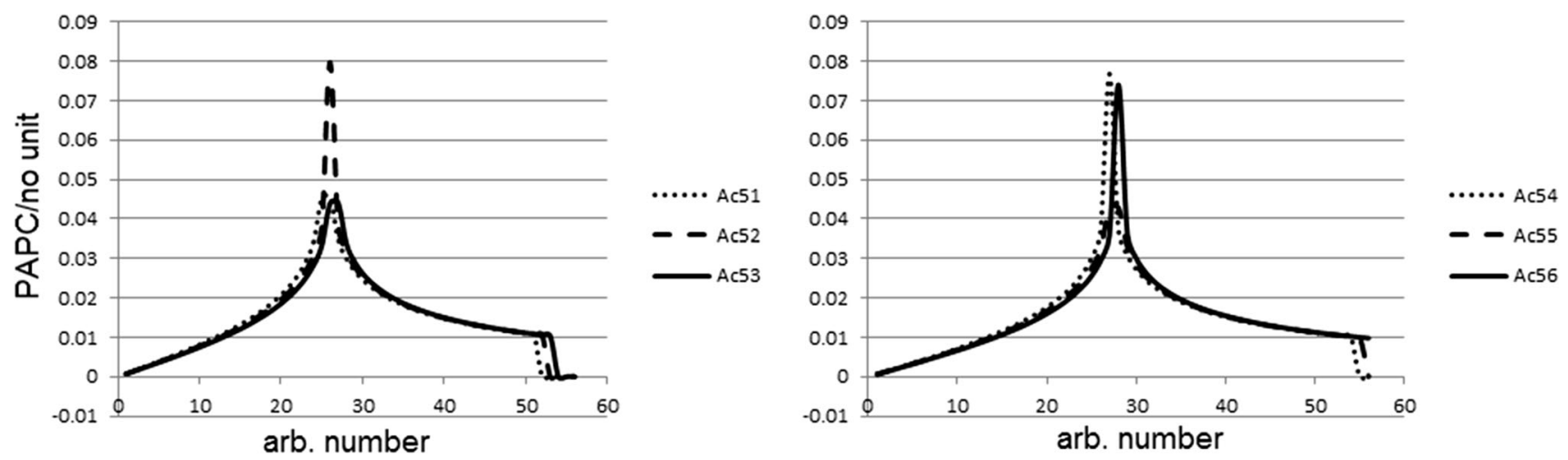

Fig. 12 PAPCs $\left(A_{c}\right)$ for atoms with atomic numbers $z=51-56$
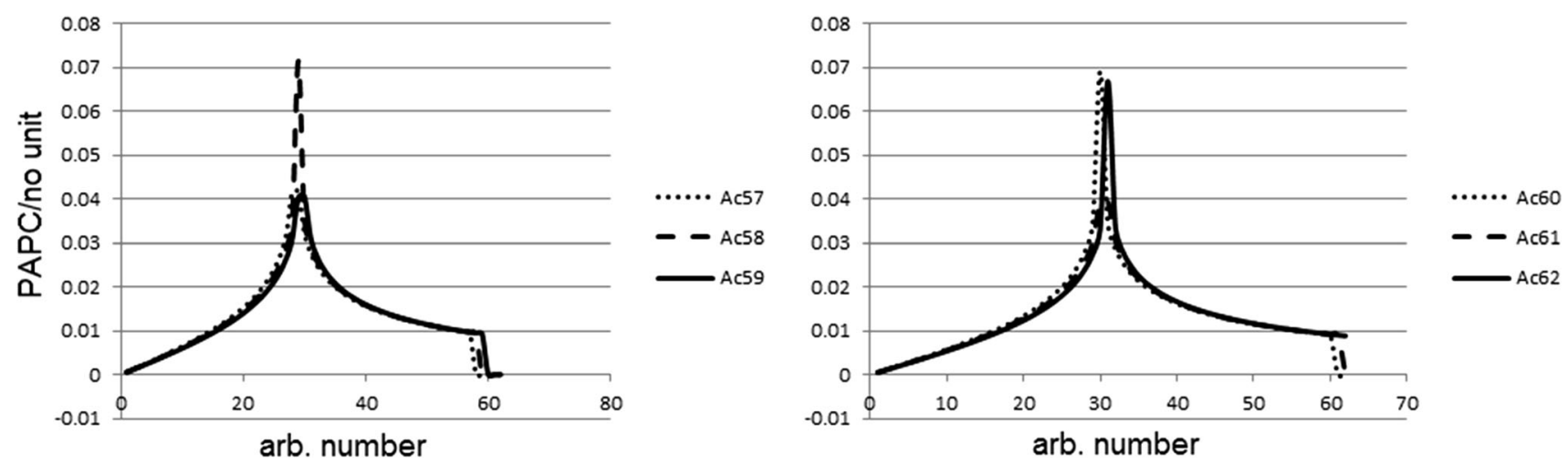

Fig. 13 PAPCs $\left(A_{c}\right)$ for atoms with atomic numbers $z=57-62$
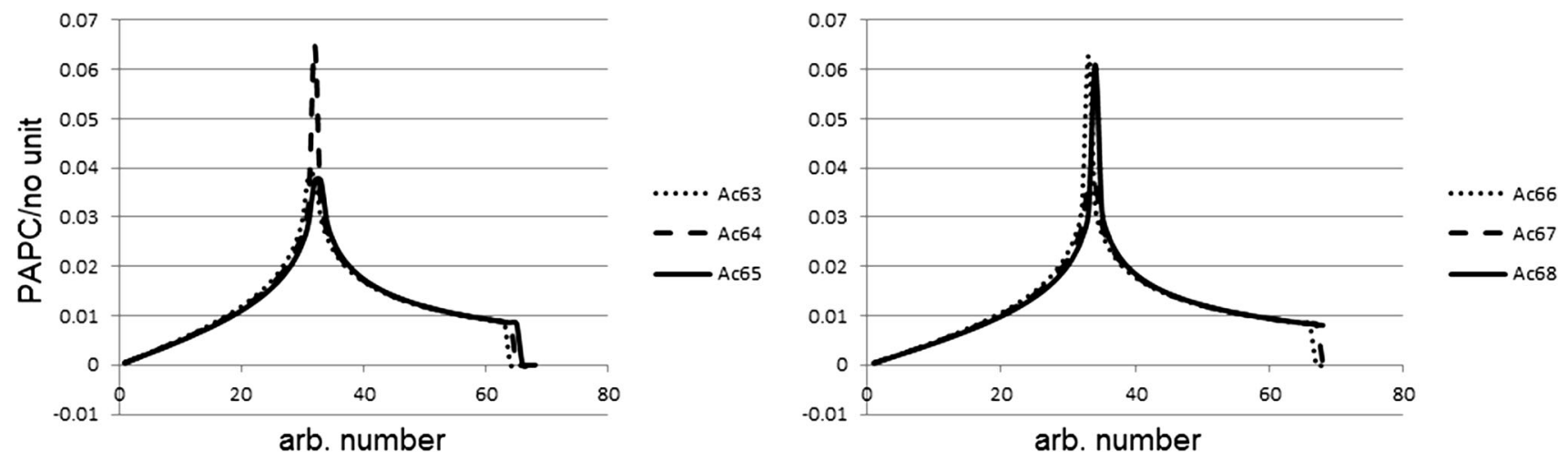

Fig. 14 PAPCs $\left(A_{c}\right)$ for atoms with atomic numbers $z=63-68$

into how to separate the PAPCs of materials with very close ones. For each case, we studied its likely behavior and reported our findings.

The little spike at mid-points of all PAPC is due to some irregularities in the atomic system which is more for the even $z$ atoms than the odd $z$ atoms, as our results show. If, for instance, we have more than one atoms in a material compound such as water $\mathrm{H}_{2} \mathrm{O}$ or oxygen $\mathrm{O}_{2}$ or ozone $\mathrm{O}_{3}$, we then have double of PAPC for the oxygen atom in $\mathrm{H}_{2} \mathrm{O}$.
Similarly, ozone will be three times the PAPC of oxygen. Therefore, if we are to explain, using the application of the PAPC, why $\mathrm{H}_{2} \mathrm{O}$ is a liquid but ozone $\mathrm{O}_{3}$ being a gas, we then say that the $\mathrm{O}_{2}$ in $\mathrm{H}_{2} \mathrm{O}$ have lower PAPC value than oxygen. Furthermore, if we count the number of joints in a PAPC plot, and if a PAPC joint is of high PAPC value, then it means that material will absorb higher photon because of the higher PAPC values. We found out that a unique material could be formed, as in the cases of $\mathrm{S}_{6} \mathrm{~F}$ and 

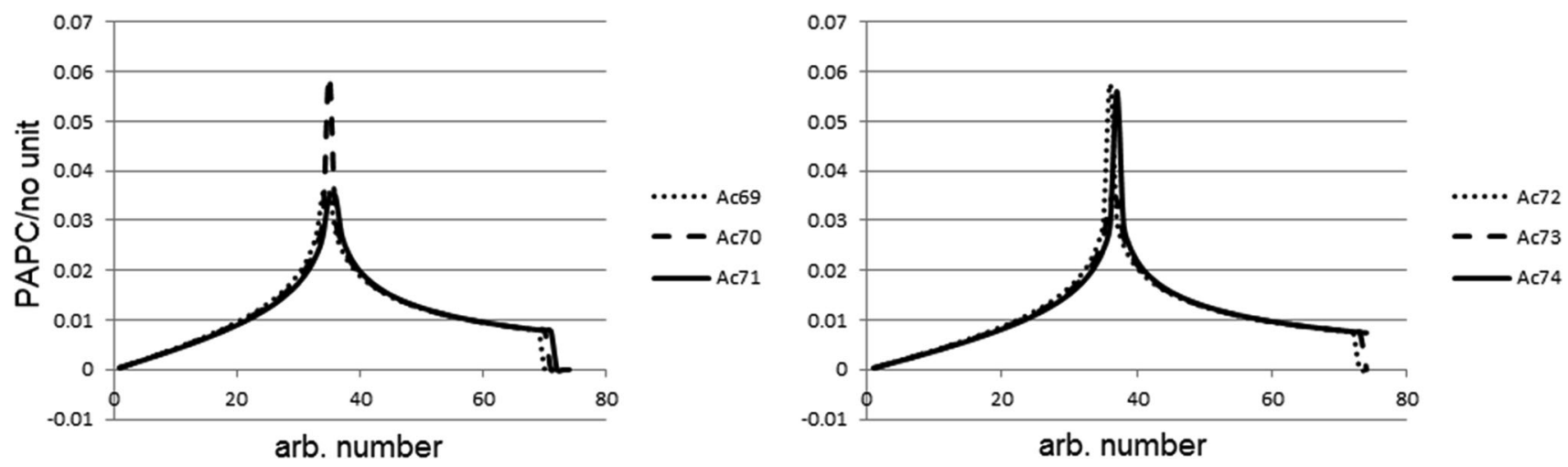

Fig. 15 PAPCs $\left(A_{c}\right)$ for atoms with atomic numbers $z=69-74$
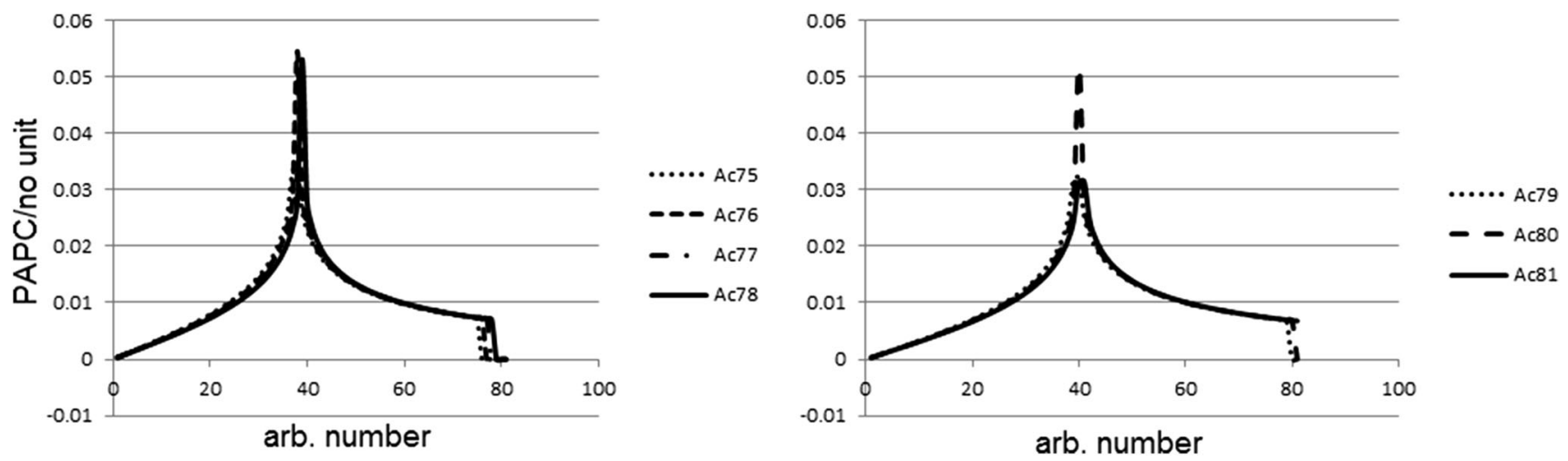

Fig. 16 PAPCs $\left(A_{c}\right)$ for atoms with atomic number $z=75-81$

Table 1 Comparing the PAPCs of each element on the periodic table

\begin{tabular}{lcl}
\hline Figure & PAPC & Difference \\
\hline Figure 4 & $0.16-0.23$ & 0.07 \\
Figure 6 & $0.075-0.12$ & 0.045 \\
Figure 8 & $0.05-0.076$ & 0.026 \\
Figure 10 & $0.041-0.059$ & 0.018 \\
Figure 12 & $0.034-0.047$ & 0.013 \\
Figure 14 & $0.029-0.039$ & 0.01 \\
Figure 16 & $0.025-0.034$ & 0.009 \\
Figure 5 & $0.1-0.15$ & 0.05 \\
Figure 7 & $0.06-0.09$ & 0.03 \\
Figure 9 & $0.045-0.065$ & 0.02 \\
Figure 11 & $0.038-0.05$ & 0.012 \\
Figure 13 & $0.031-0.042$ & 0.011 \\
Figure 15 & $0.028-0.037$ & 0.009 \\
\hline
\end{tabular}

CHN in Figs. 20, 21, 22 and 23 respectively, when the PAPCs of all its constituent atoms, at an instance of energy, cross one another. Actually, virtually all materials will have crossed PAPCs but what determines the choice of material for certain types of performance or purpose is the energy at which the PAPCs cross (Fig. 24). Since the $x$-axis

is the number of electrons and it also means the energy of that number of electrons in the PAPC plots above, then the number of crossings at earlier (lower) numbers of PAPCs means relatively higher activity at lower temperatures and vice versa. This is evident in the many crossings for the PAPC plots of superconducting materials in Fig. 25. Hence, we can derive a "Genome" of PAPC for all, such as superconducting, materials, and biological cells like cancer cells.

\section{Application of results}

To further study the usefulnesses of the PAPC, we apply the PAPC in a potential for studying molecular dynamics perturbation on ion-sputtered surfaces, earlier derived by the authors of this paper $[10,11]$. The purpose for this application (of the PAPC in a potential) is because the potential was developed for studying 'general,' i.e., all cases of different ions striking and penetrating different surfaces [11]. Therefore, the PAPC was helpful in enabling the potential to achieve its purpose of generality. The principle for the operation of the potential is that the energy thrown off by the, gradually, approaching foreign particle captures and causes an ionization of a group of atoms on the surface. This 

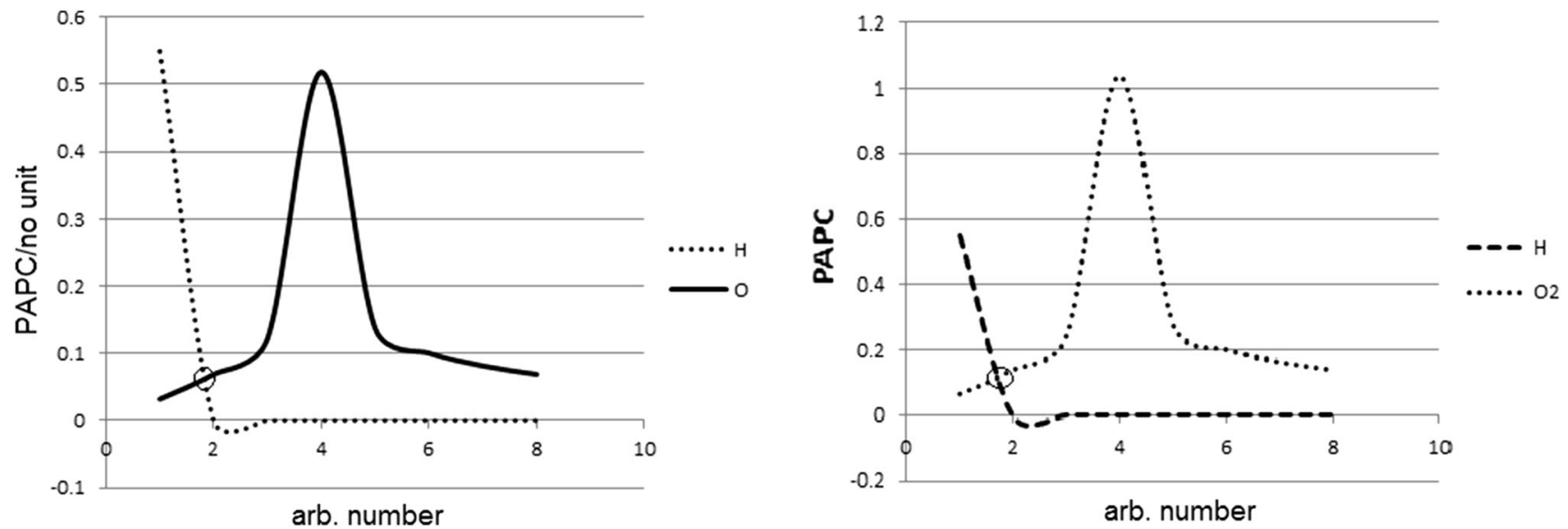

Fig. 17 Left PAPC plot for $\mathrm{OH}$. Right PAPC for $\mathrm{H}_{2} \mathrm{O}$. The molecule has its hot (active) spot at the place circled
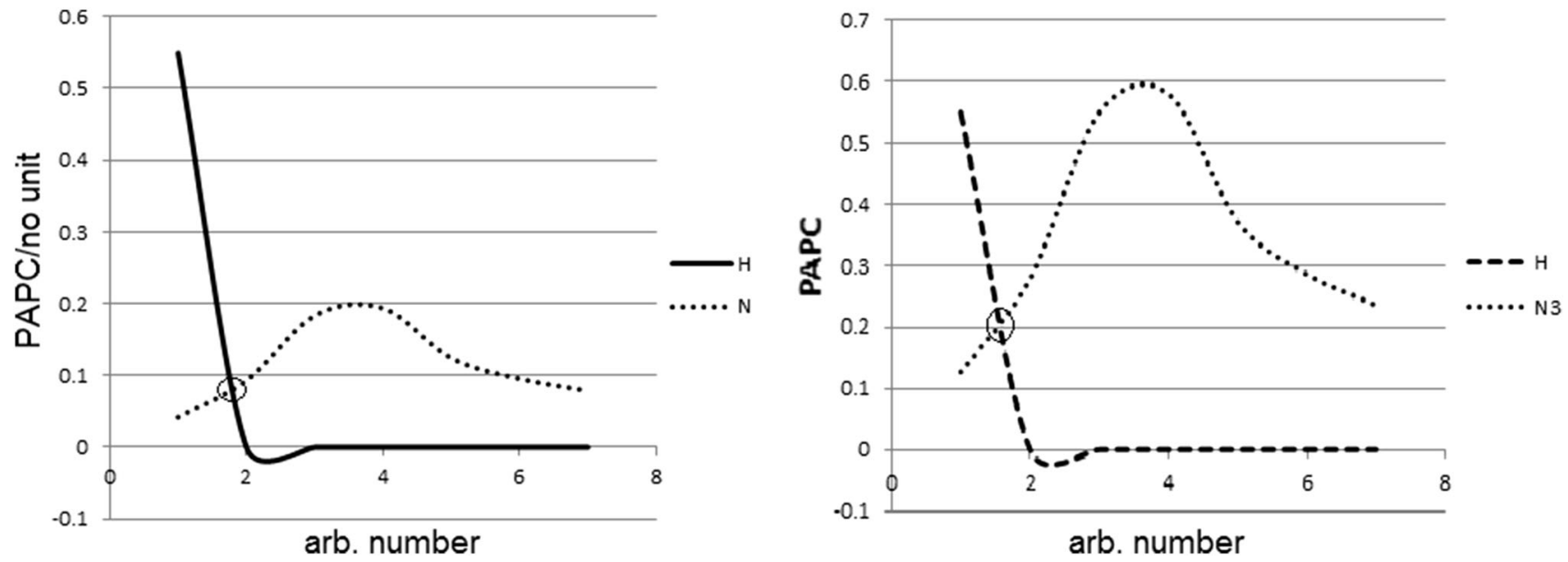

Fig. 18 Left PAPC plot for NH. Right PAPC for $\mathrm{NH}_{3}$
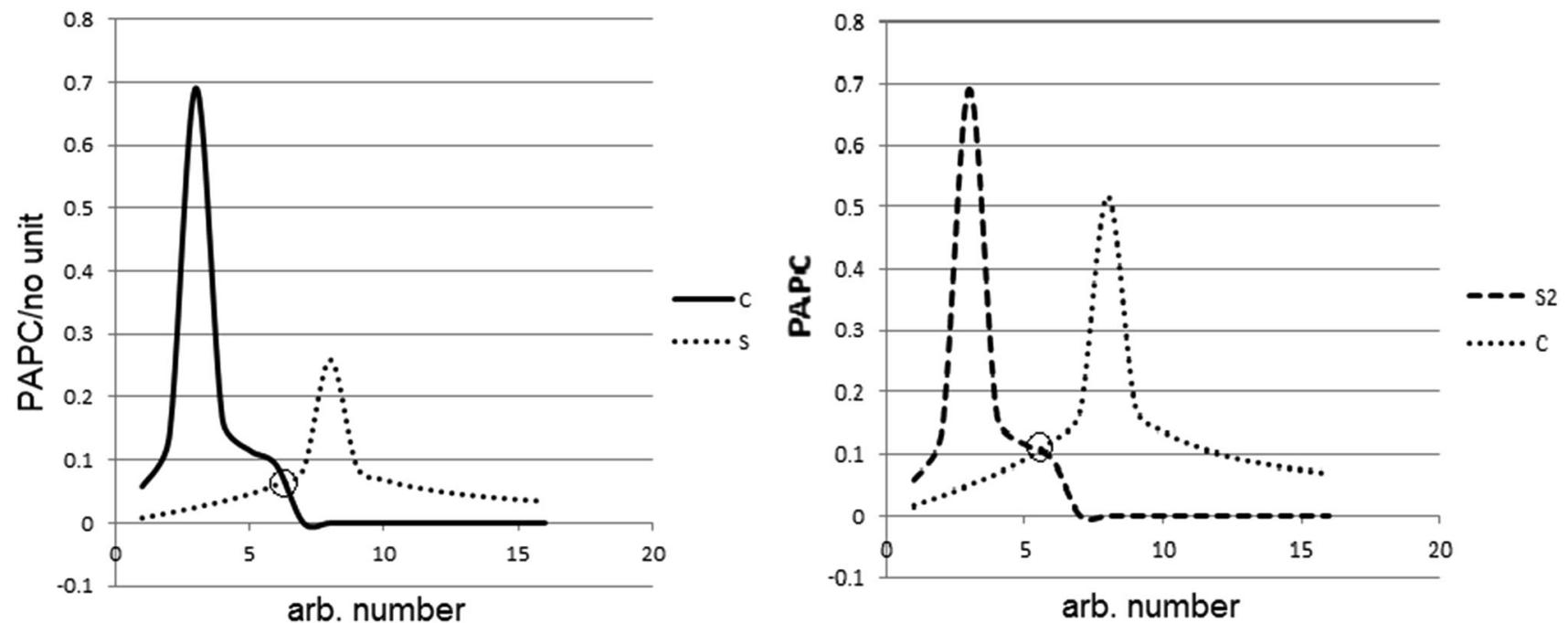

Fig. 19 Left PAPC plot for CS. Right PAPC for $\mathrm{C}_{2} \mathrm{~S}$. This plot's active site is unattainable (at room temperature), since this molecule will attain its active spot when carbon has lost or gained extra seven electrons 


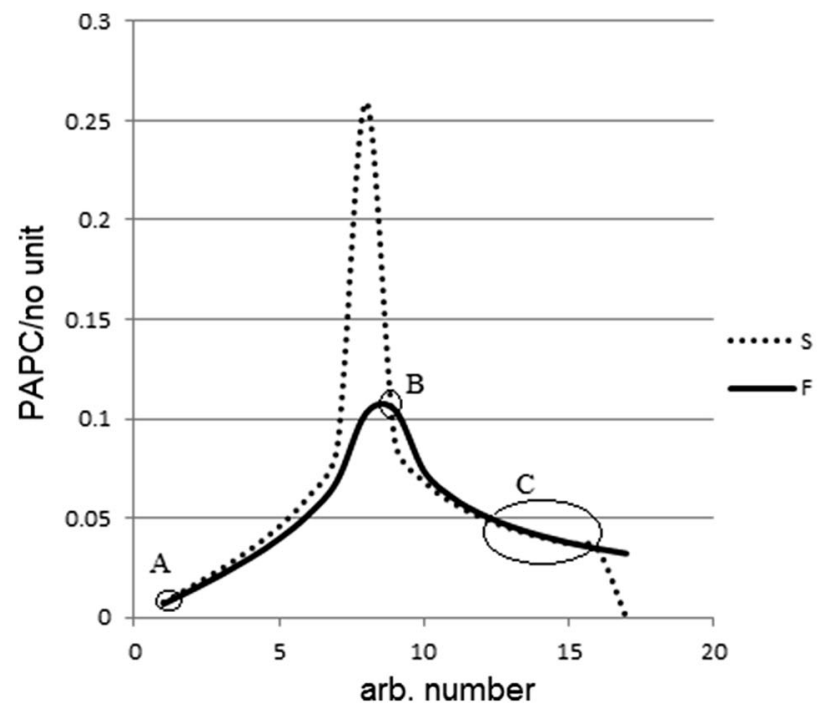

Fig. 20 Left $\mathrm{PAPC}$ plot for SF. Right $\mathrm{PAPC}$ for $\mathrm{S}_{6} \mathrm{~F}$. This suggests a very active material at $A$ the loss or gain of their first electrons, but a slightly poor material beyond gain or loss of their first electrons.

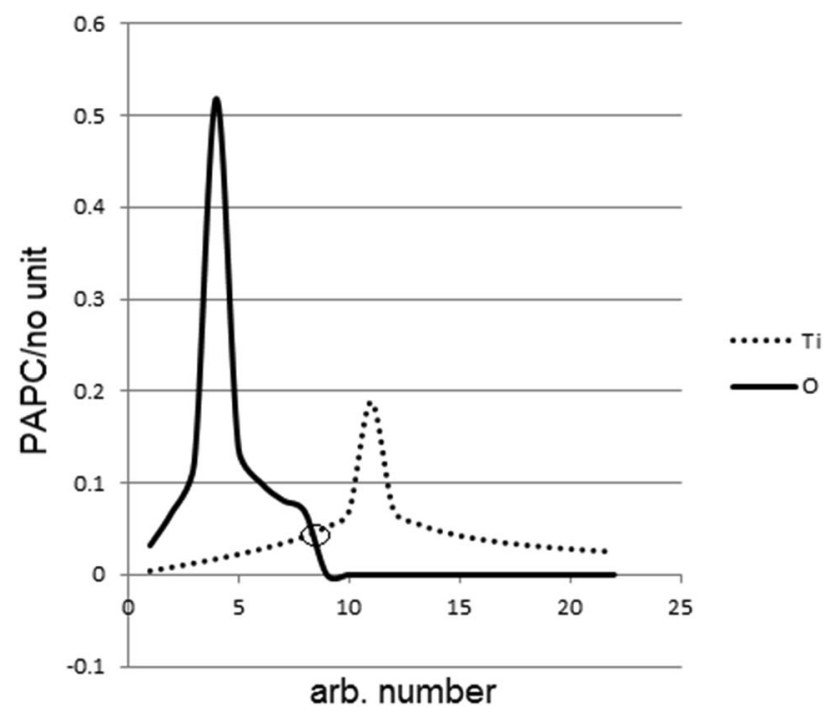

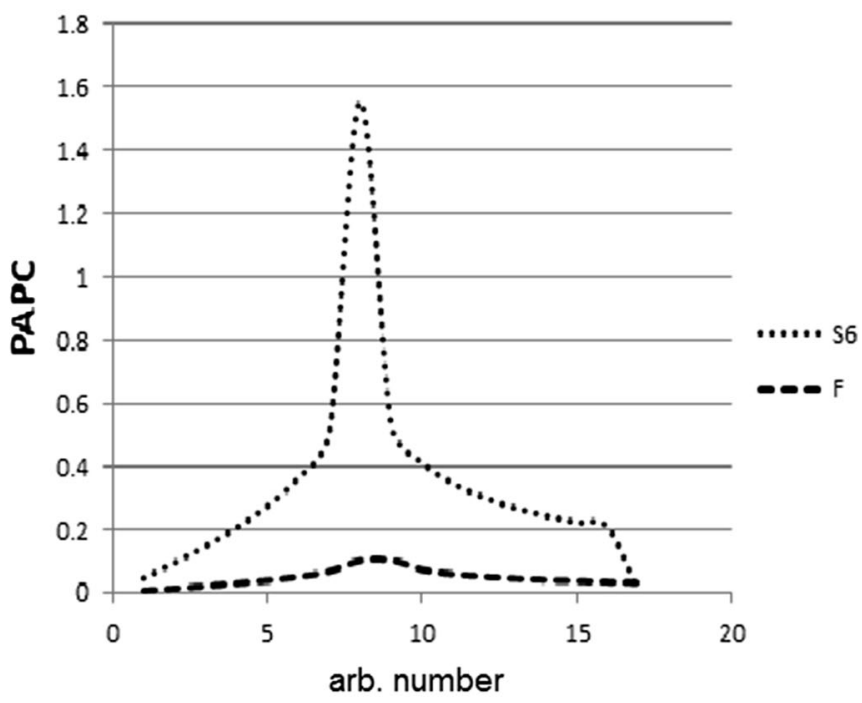

Other active spots at $B$ and $C$ might be unattainable at room temperature since it requires the loss or gain of 9 and 13 electrons respectively

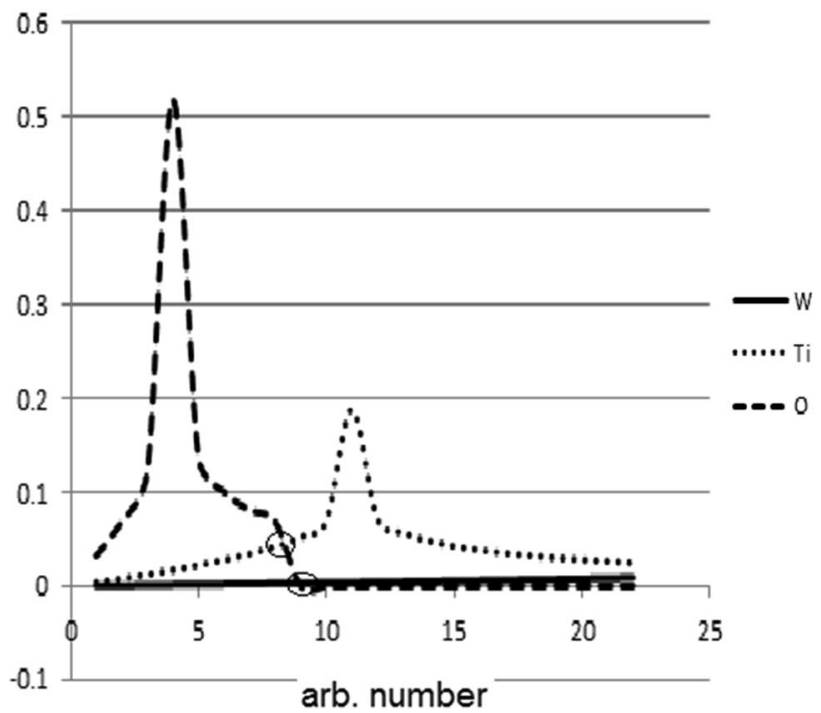

Fig. 21 Left PAPC plot for TiO. Right PAPC for WTiO. This plot's active site is unattainable at room temperature

(group) ionization leads to a build up of forceful repulsive energy amongst the captured atoms. This repulsion eventually translates to surface sputtering and penetration of the incoming particle. Therefore, this incoming particle's energy does work on the surface of the material it approaches. To this end, we introduce the proposed work done on the surface by the incoming beams of ions. Before we state the proposed work done, we state the repulsion potential, similarly to those presented in [12], suitable for the work done, and comparable to well-known potentials such as Lennard-Jones' and Morse's [13, 14], as follows:

$$
\Upsilon=\gamma\left(\mathrm{e}^{\left(\frac{1}{\pi \sigma}\right)}-\frac{\hat{\kappa} \hat{\mu}}{\gamma^{2}}\right) \Gamma(\zeta),
$$

where

- $\gamma$ is the energy of the incoming ion,

- $\sigma \rightarrow \sigma_{i+1}=\sigma_{i}+\triangle \sigma, \quad\left[\right.$ where $\left.\triangle \sigma=\exp \left(-\frac{\gamma}{\theta \sigma}\right)\right] \quad$ is the distance covered by the photon cone of the ion. If the sputtering angle, $\theta$ which can take values from 0 and $90^{\circ}$, is 0 , that is the approach on the surface is perpendicular to the surface, then we have $\triangle \sigma=0$. 
Fig. 22 PAPC plot for $\mathrm{CSiO}$. The site $A$, though not a good active site, might cause a flux of electron flow because of its close range. In essence, this molecule's activity (at room temperature) might be determined by only carbon and oxygen at site $B$

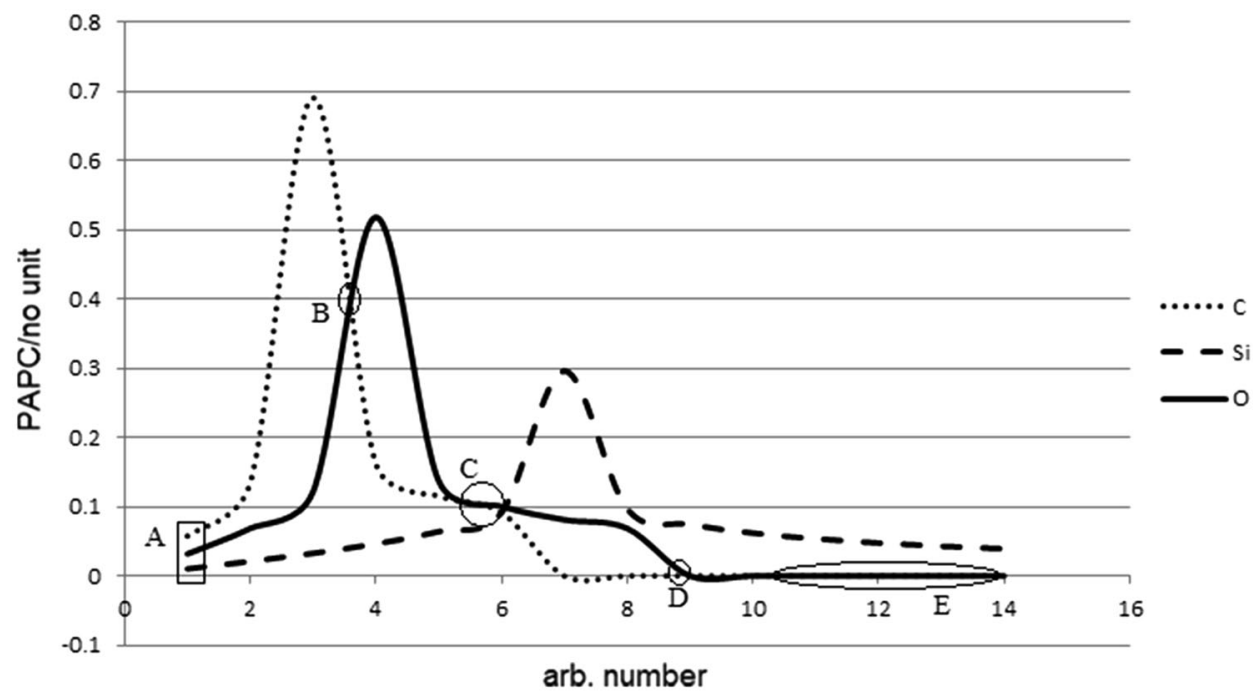

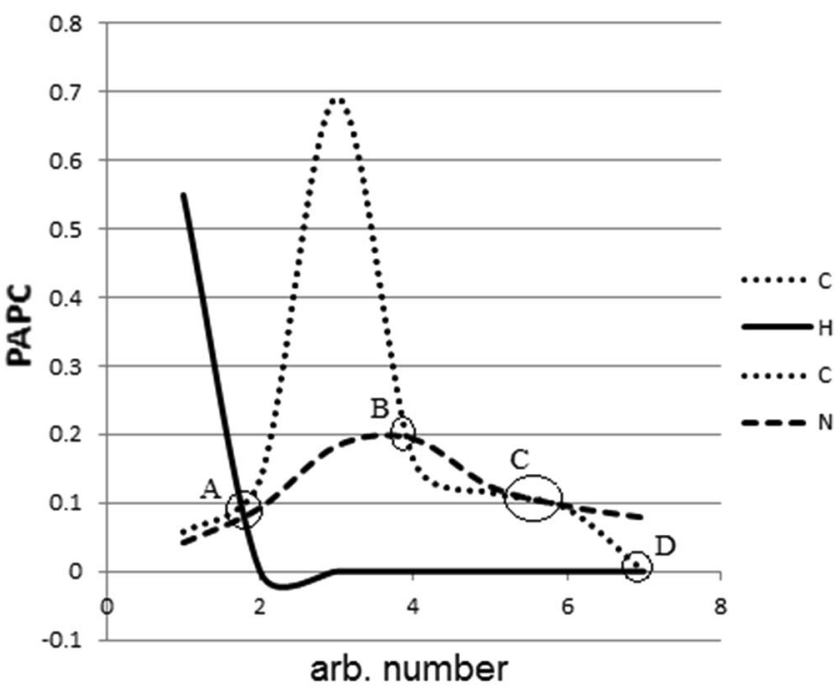

PAPC plots so far, this molecule has all its constituent atoms active at a single spot $A$. This makes the material to be very active
Fig. 23 Left PAPC plot for SiCH. Again, hydrogen plays the role of initializing interactions at sites $A$ and $B$. Right PAPC for CHN. Again, hydrogen plays the role of initializing interactions at sites $A$. Of all the

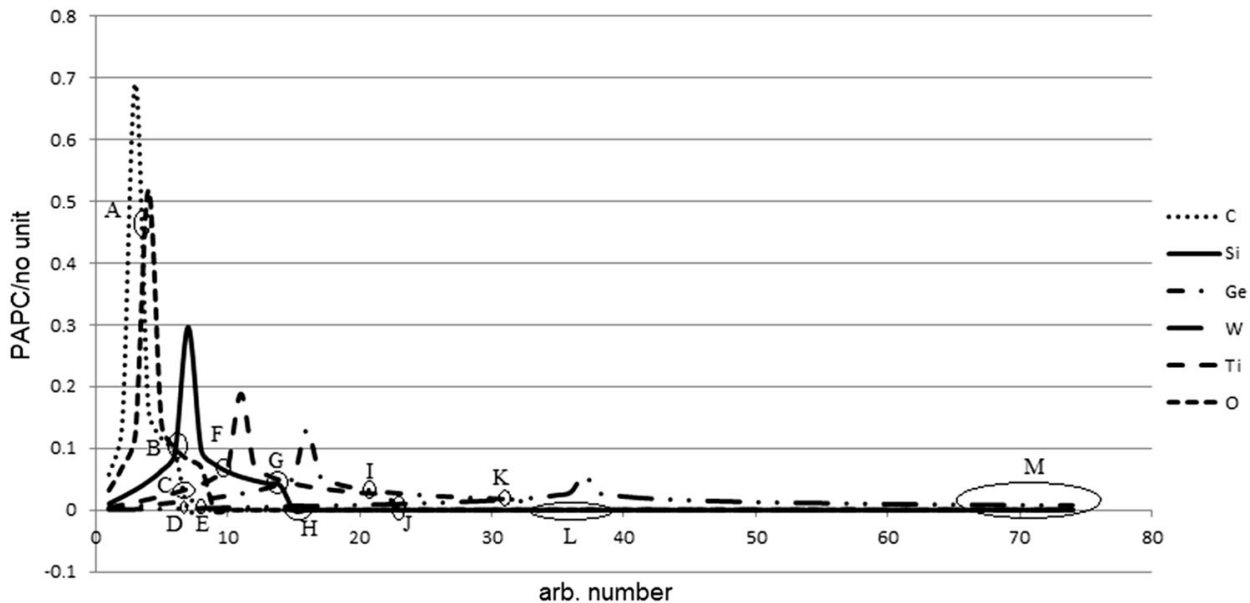



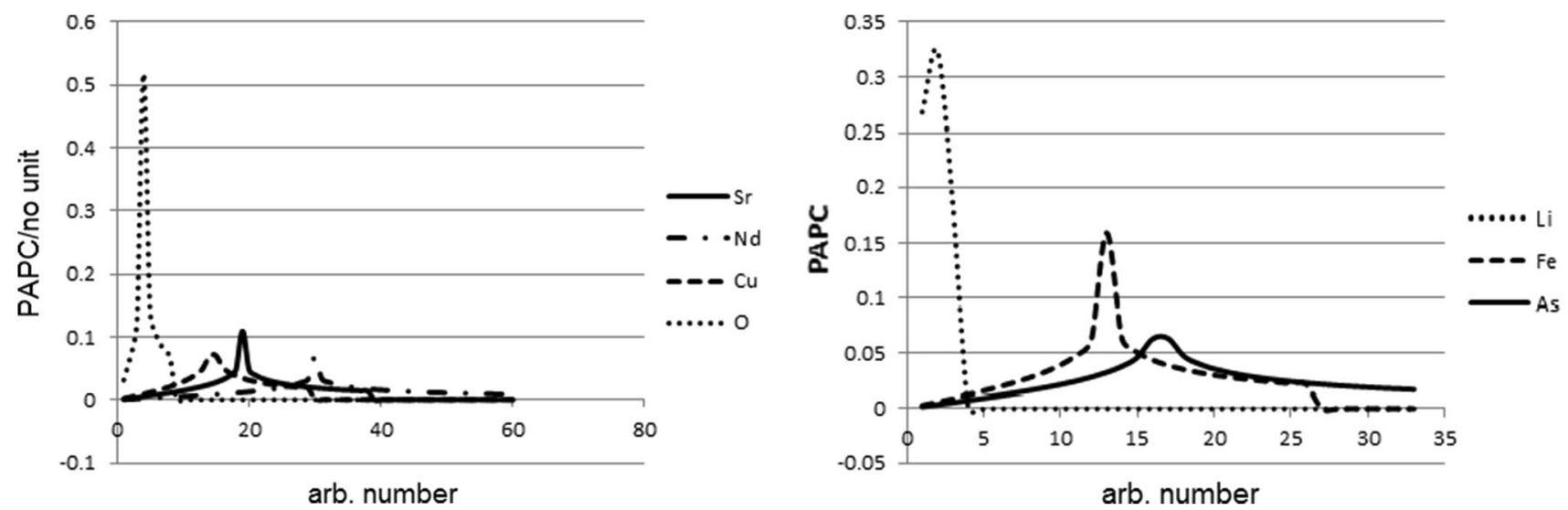

Fig. 25 Left PAPC plot for SrNdCuO (40K). Right PAPC for LiFeAs (18K)
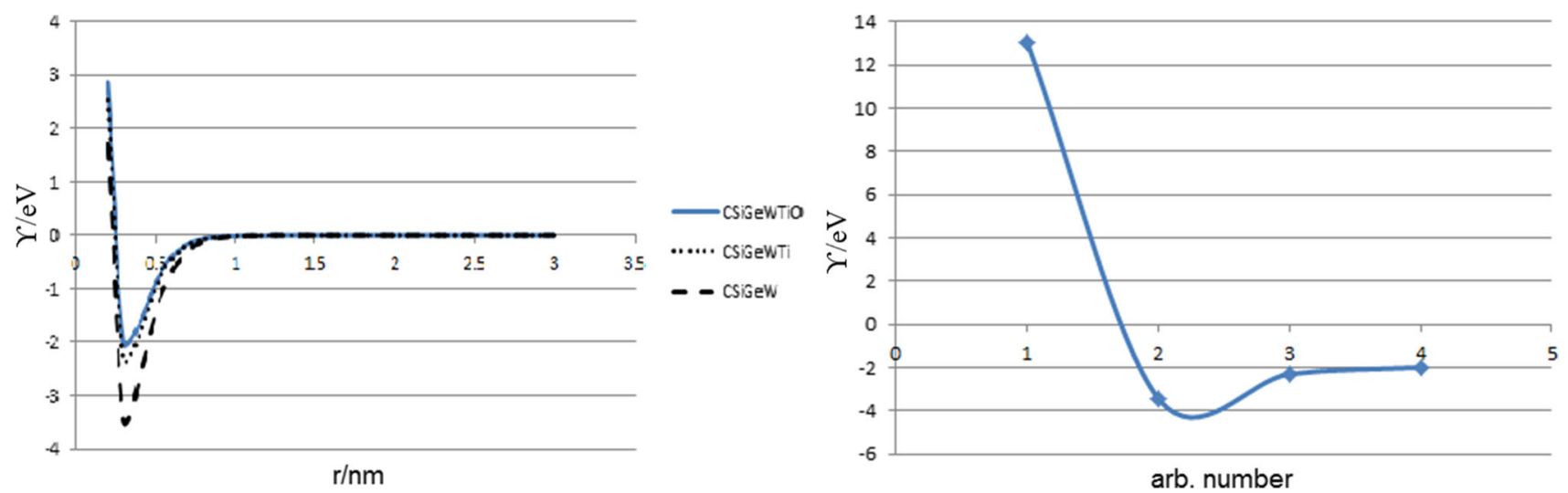

Fig. 26 Left Potential plot for CSiGeWTiO, CSiGeWTi and CSiGeW. Right Phase shift in the molecular dynamics of the changing potential in the binding process from $\mathrm{CSiGe}$ to $\mathrm{CSiGeW}$ to $\mathrm{CSiGeWTi}$ to $\mathrm{CSiGeWTiO}$ at same distance $0.3 \mathrm{~nm}$, starting with $\mathrm{CSiGe}$

$$
\begin{aligned}
\hat{\kappa} & =\frac{k \text { region }}{\sum \text { atomic diameters of atoms in } k \text { region }} \\
& =\frac{k \text { region }}{\sum D_{p q}-\cdots \sum D_{l m}-\sum D_{k l}+\sum D_{j k}-\sum D_{i j}+\sum D_{1}}
\end{aligned}
$$

is the lattice site occupation density of the topograph of the surface in which the ion is to land. Please note that the default $k$ region for a flat surface is a semicircular shape. Where $\sum D_{p q}$ is the sum of all atoms in the $N_{p q}$ population.

$\hat{\mu}=A_{c} \times$ atomicnumber

is the property of the molecules on whom the ion is to land. The properties are the PAPC and the atomic numbers of the atoms of that molecule.

$$
\zeta=\beta \hat{\mu} \sigma
$$

is the quantity that determines the activation of the sputtering as the ion is approaching the surface. $\beta$ is a constant that stabilizes the rate of increment or reduction of $\sigma$,

$$
\Gamma(\zeta)=1-\tanh (\zeta)
$$

helps to activate the sputtering process. This is because when the ion is still far off, above the surface, there will not be sputtering but as it approaches the surface, the sputtering process starts to build, gradually.

Therefore, we may wish to write:

$\Upsilon=\gamma\left(\mathrm{e}^{\left(\frac{1}{\pi \sigma}\right)}-\frac{\hat{\mu} \int \mathrm{d}^{3} \sigma}{\gamma^{2} \sum_{i} D_{i}}\right) \Gamma(\zeta)$,

where $D_{i}$ are the diameters of the atoms in the $k$ region. 

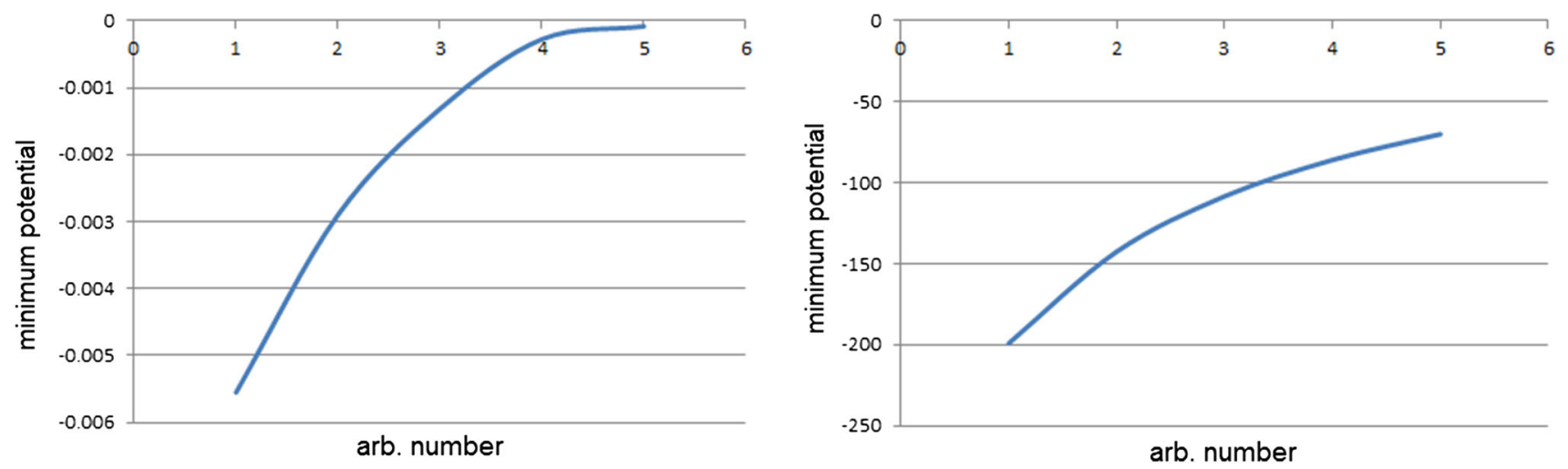

Fig. 27 Left Minima potential plot for $\mathrm{Au}+\mathrm{O}, \mathrm{Au}+2 \mathrm{O}, \ldots, \mathrm{Au}+5 \mathrm{O}$. Right Minima potential plot for $\mathrm{Fe}+\mathrm{O}, \mathrm{Fe}+2 \mathrm{O}, \ldots, \mathrm{Fe}+5 \mathrm{O}$

Finally, we now have the proposed work done on the surface, by one ion's photon shower, to be given as:

$$
\begin{aligned}
\Omega= & \sigma \frac{\mathrm{d} \Upsilon}{\mathrm{d} \sigma} \\
\Omega= & \gamma \exp \left(\frac{1}{\pi \sigma^{2}}\right)-\frac{\sigma \hat{\mu} \partial_{\sigma} \int \mathrm{d}^{3} \sigma}{\gamma^{2} \sum_{i} D_{i}} \\
& -\left(\gamma \exp \left(\frac{1}{\pi \sigma^{2}}\right)-\frac{\sigma \hat{\mu} \partial_{\sigma} \int \mathrm{d}^{3} \sigma}{\gamma^{2} \sum_{i} D_{i}}\right) \tanh (\beta \hat{\mu} \sigma) \\
& -\beta \hat{\mu} \sigma\left(\gamma \exp \left(\frac{1}{\pi \sigma^{2}}\right)+\frac{\hat{\mu} \partial_{\sigma} \int \mathrm{d}^{3} \sigma}{\gamma^{2} \sum_{i} D_{i}}\right) \operatorname{sech}^{2}(\beta \hat{\mu} \sigma) .
\end{aligned}
$$

Now, we have shown, in our results, that there are noticeable changes in the minimum vibration, or potentials; for different phases of the molecules' interaction with its environment, we shall present in the following the figures to confirm our investigation. Figure 26 indicates the observed changes in the subsequent interactions that formed material CSiGeWTiO. In this material, we started out by assuming that the initial composition was just CSiGe. Then, $\mathrm{W}$ interacts with it to give $\mathrm{CSiGeW}$ and then $\mathrm{Ti}$ and $\mathrm{O}$, in turn, to give CSiGeWTiO. In each case, the observed change in the minimum potentials, at an equilibrium distance of $0.3 \mathrm{~nm}$, is shown in the left part of the figure.

In another example of PAPC applications, we present our findings (as in Fig. 27), as regards molecular binding processes of gold and iron with first, second, and up to the fifth oxygen atom. This result suggests that gold material is irritated by more and more oxygen atoms interacting with it [15]. As shown in Fig. 27, both iron and gold materials seem to terminate in their continuing interactions with oxygen atoms (in their environment) but gold terminates far more quickly than iron.

From both examples, we conclude that there is a need to study the molecular dynamics (with the aid of PAPC calculations) as well as the surface approach of the environmental atoms (or particles) as a whole. This is to have a full understanding of the manner in which materials execute molecular dynamics. This is a clear evidence that even the molecular dynamics is changing (and thus the PAPC) over time while the material acquires history. The knowledge of PAPC would thus help in simulating the various perturbations of molecular dynamics over time.

Open Access This article is distributed under the terms of the Creative Commons Attribution 4.0 International License (http://crea tivecommons.org/licenses/by/4.0/), which permits unrestricted use, distribution, and reproduction in any medium, provided you give appropriate credit to the original author(s) and the source, provide a link to the Creative Commons license, and indicate if changes were made.

\section{References}

1. Lang, P.F., Smith, B.C.: Methods of calculating ionization energies of multielectron (five or more) isoelectronic atomic ions. Sci. World. J. 2013, Article ID 157412 (2013). doi:10.1155/2013/157412

2. Clarage, J.B., Romo, T., Andrews, B.K., Pettitt, B.M., Phillips Jr., G.N.: A sampling problem in molecular dynamics simulations of macro-molecules. Proc. Natl. Acad. Sci. USA Biophys. 92(4), 3288-3292 (1995)

3. Marletta, Giovanni, Catalano, Salvatore M., Pignataro, Salvatore: Chemical reactions induced in polymaers by kev ion, electrons and photons. Surf. Interf. Anal. 16(1-12), 407-411 (1990). doi:10.1002/sia.740160185

4. Balogh, A.G., Baba, K., Cohen, D.D., Elliman, R.G., Ensinger, W., Gyulai, J.: Modification, synthesis, and analysis of advanced materials using ion beam techniques. In: Advances in Materials Science and Engineering, vol. 2012. Hindawi Publishing Corporation, Article ID 431297. doi:10.1155/2012/431297

5. Gottberg, A.: Target materials for exotic ISOL beams. In: Nuclear Instruments and Methods in Physics Research Section B: Beam Interactions with Materials and Atoms. doi:10.1016/j.nimb. 2016.01.020

6. Brumer, P., Shapiro, M.: Molecular response in one-photon absorption via natural thermal light vs. pulsed laser excitation. PNAS 109(48), 19575-19578 (2012). http://www.pnas.org/cgi/ doi/10.1073/pnas.1211209109

7. Lide, D.R.: Handbook of Chemistry and Physics, 84th edn, vol. 10. CRC Press, USA (2003) 
8. Riley, K.F., Hobson, M.P., Bence, S.J.: Mathematical Methods for Physics and Engineering. Cambridge University Press, Cambridge (2002)

9. Gauthier, R.: The electron is a charged photon with the de Broglie wavelength. Santa Rosa Junior College. http://www.super luminalquantum.org

10. Akande, R.O., Oyewande, E.O.: Theoretical monitoring of energy transport on solid surfaces at nano-metric scales. arXiv:1511. 09384 [cond-mat.mtrl-sci]

11. Akande, R.O., Oyewande, E.O.: A general potential for molecular dynamics of ion-sputtered surfaces. arXiv:1510.02791 [physics.chem-ph]
12. Saitoh, K., Hayakawa, H.: Simulation of depositions of a Lennard-Jones cluster on a crystalline surface. Progr. Theor. Phys. 122(5) (2009)

13. Van Hung, N.: A method for calculation of Morse potential for fcc, bcc and hec crystals applied to Debye-Waller factor and equation of state. Commun. Phys. 14(1), 7-14 (2004)

14. Frank, M., Mourits, M., Rummens, F.H.A.: A critical evaluation of Lennard-Jonnes and Stock Mayer potential parameters and some correlation methods. Can. J. Chem. 55, 3007 (1997)

15. Aqua Regia. Gold and treasure webpage (2015). http://www. goldandtreasure.com/Aquaregia.htm 